\title{
OP-45 STRENGTHENING THE MOTHER AND CHILD TRACKING SYSTEM THROUGH AN ENHANCED REVIEW MECHANISM USING A SIMPLE TOOL
}

SM Sadhana. KSHSRC - Karnataka State Health System Resource Centre, Bangalore (Karnataka), India

\subsection{6/bmjgh-2016-EPHPabstracts.45}

Background Mother and Child Tracking System (MCTS) is a guiding tool to assess maternal and child health services. MCTS is a centralised web-based application that helps tracks beneficiaries (pregnant women and children up to five years of age) and monitors maternal and child health service delivery. The Karnataka State Health System Resource Centre (KSHSRC) has developed a user-friendly tool to update MCTS. By using this tool - a single spreadsheet per sub-centre $-\mathrm{a}$ line list of all the antenatal cases and immunisation services is generated. The Thaayi Card (mother card issued to antenatal cases) number is used as the identification number for each beneficiary. The use of this single sheet simplifies the work of field health workers significantly while updating MCTS. Along with the 'due lists', cohort reports for antenatal services and child immunisation services are also generated by this tool, which are used for review of reproductive and child health programme at sub-health-centre level up to the district level. This initiative has 


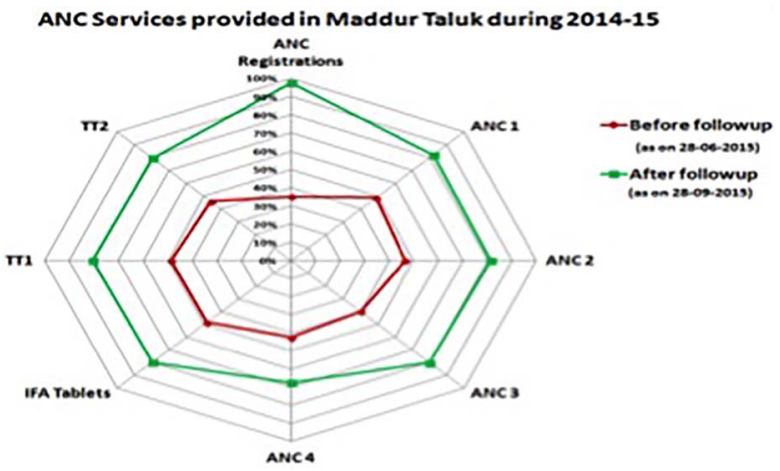

Abstract OP-45 Figure 1 Antenatal care services, Maddur taluk, Karnataka, 2014-2015.

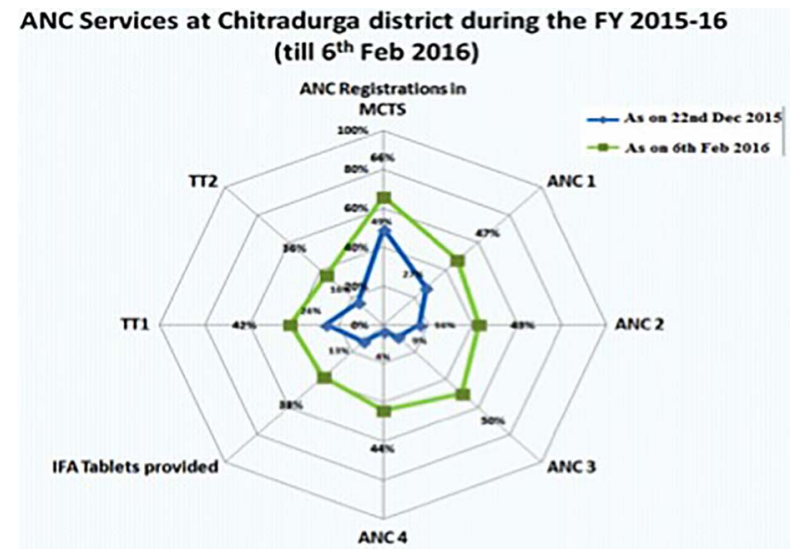

Abstract OP-45 Figure 2 Antenatal care services, Chitradurga district, Karnataka, 2015-2016.

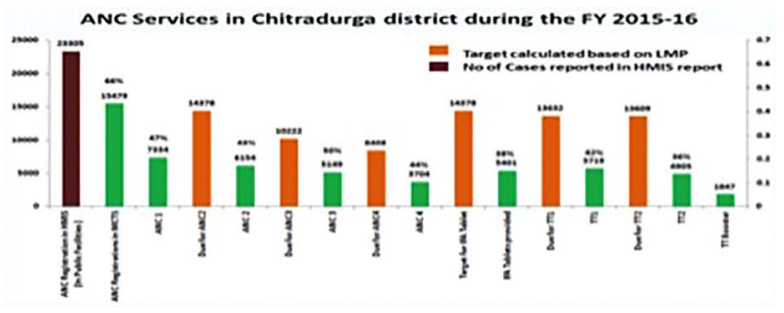

Abstract 0P-45 Figure 3 Cohort report for antenatal care services, Chitradurga district, 2015-2016.

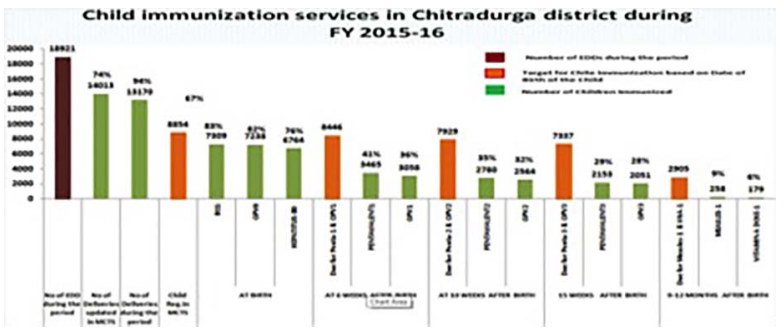

Abstract OP-45 Figure 4 Cohort report for child immunisation services, Chitradurga district, 2015-2016. been taken up in 396 sub health centres within jurisdiction of 98 primary health centres of two districts in Karnataka.

Methods The resource team of the KSHSRC conducted orientation workshops to sensitise the concerned officials about the tool and its usage at the identified districts and sub-districts. Hands-on trainings were also provided to relevant staff at primary health centres including the medical officers. Several follow-up visits were made to ensure proper implementation and to review progress along with concerned staff.

Findings Remarkable improvements have been documented in updating MCTS and review mechanisms. We present the comparison of the status of MCTS before and after the use of this tool. As shown in figures 1 and 2, antenatal case registration updates in MCTS improved remarkably. In Maddur taluk (subdistrict), in 2014-2015, antenatal case registration improved from $35 \%$ to $98 \%$ in the span of about three months. Similarly, in Chitradurga district, in 2015-2016, antenatal case registration improved from $49 \%$ to $66 \%$ within a span of about one and a half months.

In order to strengthen the review mechanisms, cohort reports were generated for antenatal care services (see figure 3) and child immunisation services (see figure 4).

These cohort reports ease and simplify the review mechanism from sub-health-centre up to district level.

Discussion By improving data availability in MCTS, the implementation of maternal and child health services can be effectively tracked for timely action by implementers. This would lead to reduction in preventable maternal deaths. 